\title{
Emociones morales en educación: análisis del enfado en el contexto neoliberal
}

Moral emotions in education: analysis of anger in the neoliberal context

\section{Felipe Nicolás Mujica Johnson ${ }^{1}$}

\section{Resumen}

Desde la Antigua Grecia se ha generado un discurso que desprecia las emociones y las relega al ámbito personal. Pero, hace años, ese discurso se ha debilitado y el ámbito emocional está siendo reconocido como un aspecto relevante y, también, se ha posicionado en la vida pública. En este sentido, el presente ensayo analiza la emoción de enfado en el contexto neoliberal y algunas de sus implicancias en la educación. Para ello, se consideran diferentes aportes teóricos que exponen los beneficios culturales que suscita dicha emoción. Asimismo, se revisan algunas ideologías que favorecen la ignorancia o desvalorización del enfado en los procesos educativos. En suma, se concluye que una educación crítica debe clarificar el potencial del enfado para la transformación de la sociedad.

Palabras clave: Emoción; formación moral; justicia social; derechos humanos; políticas educativas neoliberales.

\footnotetext{
${ }^{1}$ Licenciado en Educación, Profesor de Educación Física y Magíster en Ciencias de la Actividad Física y del Deporte, Universidad de Playa Ancha de Ciencias de la Educación, Chile. Doctorando en Ciencias de la Actividad Física y del Deporte, Universidad Politécnica de Madrid, España. Email: fmujica@live.cl
} 


\begin{abstract}
From Ancient Greece a discourse has been generated that despises emotions and relegates them to the personal sphere. But, years ago, this discourse has weakened and the emotional sphere is being recognized as a relevant aspect and, also, has positioned itself in public life. In this sense, this essay analyzes the emotion of anger in the neoliberal context and some of its implications in education. For this, different theoretical contributions are considered that expose the cultural benefits that this emotion provokes. Likewise, some ideologies that favor ignorance or devaluation of anger in educational processes are reviewed. In sum, it is concluded that a critical education must clarify the potential of anger for the transformation of society.
\end{abstract}

Keywords: Emotion; moral formation; social justice; human rights; neoliberal educational policies.

\title{
1. Introducción
}

Este ensayo, con finalidades educativas, aborda las emociones de las personas desde una perspectiva integral, o sea, no las reduce a ámbitos académicos aislados, como puede ser el psicológico, biológico, filosófico o sociológico. Sino que, dado que la pedagogía es una de las artes más holísticas que nuestra memoria histórica conoce, se entiende que todos esos ámbitos de alguna manera inciden en la dimensión emocional y, por ende, en los procesos pedagógicos. Cuando se alude al carácter holístico de la educación, se hace referencia a la transversalidad de esta actividad en la sociedad, o sea, que no se reduce a una actividad profesional, ya que la mayoría de las personas en algún momento podrán ejercer una labor pedagógica como padres, madres, amigos o compañeros de trabajo. Esto no significa que no sea necesaria la especialización en el ámbito pedagógico y, en consecuencia, las profesiones en torno a la pedagogía. Savater (1997), en sus reflexiones sobre el arte de educar, plantea las siguientes ideas que pueden ser representativas del carácter holístico de la educación:

La función de la enseñanza está tan esencialmente enraizada en la condición humana que resulta obligado admitir que cualquiera puede enseñar, lo cual 
por cierto suele sulfurar a los pedantes de la pedagogía que se consideran al oírlo destituidos en la especialidad docente que creen monopolizar. Los niños, por ejemplo, son los mejores maestros de otros niños en cosas nada triviales, como el aprendizaje de diversos juegos (Savater, 1997, p. 48).

Por lo tanto, cabe destacar que, sin intención de generar algún reduccionismo, se analizará un ámbito de la dimensión emocional, en concreto, su aspecto moral en el marco de políticas educativas mercantilistas carentes de un fuerte sentido ético humanista y de justicia social (Manrique, 2015; McLaren, 2015; Monarca, 2016; Mujica e Inostroza, 2020). El ámbito moral hace referencia a los hábitos y costumbres de las personas (Durán, 2011), y en este escrito se asociará a los valores éticos que la podrían configurar. Entre esos valores encontramos la solidaridad, la justicia, el respeto, la humildad y la responsabilidad (Savater, 1997; Scheler, 2010). Otros valores, y disvalores, característicos de las sociedades capitalistas, son la libertad, el egoísmo, la individualidad y la competitividad (McLaren, 2015; Scheler, 2010). Con base en lo mencionado anteriormente, este ensayo tiene por objetivo analizar la emoción de enfado en el contexto neoliberal y algunas de sus implicancias en la educación.

Para clarificar el concepto de emoción, se ha considerado pertinente incluir dos significados. En primer lugar, el de Alonso (2017), quien señala que emoción es "una respuesta afectiva intensa y breve que se produce en respuesta a un suceso o una situación determinada, y que va asociada a cambios corporales específicos y está relacionada con lo que es importante para nosotros" (p. 17). En segundo lugar, se encuentra el de Puig (2012), quien, desde una perspectiva sociológica, plantea la siguiente conceptualización:

La emoción no es una simple reacción fisiológica, sino que, a pesar de tener una dimensión de este tipo, también está relacionada con rasgos de personalidad (dimensión psicológica), subculturas específicas (modalidades deportivas -en nuestro caso-, género, edad...) y contextos socioculturales e históricos (Puig, 2012, p. 106).

Precisamente, la pertinencia de dichos significados de emoción responde a su apertura a diferentes ámbitos de estudio al reconocer el aspecto biológico, 
psicológico y social. Cabe mencionar que, en este ensayo, el análisis se centrará en el componente subjetivo y sociocultural de la dimensión emocional. En este sentido, por una parte, las reflexiones versarán sobre algunos vínculos de aquella dimensión con preferencias morales, y éticas, que pueden adoptar los sujetos. Por otra parte, aunque no exentos de significados morales, tratarán la relación afectiva con algunas problemáticas con las que se enfrenta la sociedad actual. Y, por supuesto, con sus ideologías políticas inmersas, aprovechando los postulados que ha aportado la pedagogía crítica. Al respecto, cabe aclarar que, según Giroux (2013), "las ideologías no son solo una constelación de ideas, estereotipos, y modos de sentido común; también representan formas específicas de conocimiento y creencias arraigadas en fuertes cargas emocionales" (p. 22).

A propósito de miradas reduccionistas en torno a las emociones del ser humano, es preciso saber que la historia del pensamiento filosófico occidental ha estado marcada por aquellas concepciones que se han encargado de delimitar la afectividad. Al respecto, con la finalidad de reflejar algunas de las divergencias filosóficas sobre el tema, Casado y Colomo (2006) han manifestado que algunas personalidades han estado a favor de buscar la armonía emocional; de promover solo las emociones que suscitan placer, lo cual, evidentemente, responde al enfoque hedonista; de asumir una postura precavida frente a la experimentación de emociones 0 , en otras palabras, de moderación; $y$, por otra parte, quienes declararon una batalla contra las emociones $y$, por consiguiente, promovieron ignorarlas y rechazarlas. En términos generales, Zaccagnini (2008) plantea un progreso histórico en la interpretación de las emociones en función de cuatro contextos culturales. El primero es el de la Grecia Clásica, donde se sostenía que eran impulsos incontrolables atribuidos a los dioses; el segundo es el de la Edad Media, caracterizadas por impulsos negativos que perjudican el progreso moral $y$, en consecuencia, el camino al cielo, siendo atribuidas a las situaciones mundanas, al diablo, a los demonios y a la materia carnal; el tercero es el de la Modernidad, donde son denominadas impulsos absurdos o irracionales que amenazan la cordura o la lógica en la vida de las personas; y, por último, manifiesta que en la época actual, denominada posmodernidad, se les reconoce como estados psíquicos que 
contienen un significado producto del proceso interactivo entre el pensar, el emocionarse y el vivir en un entorno social concreto.

A pesar de que la posmodernidad nos acerca a nuevas interpretaciones del ámbito emocional en la sociedad y, en efecto, en el ámbito educativo, las emociones siguen interpretándose de forma acrítica desde perspectivas hedonistas, idealistas, psicologistas, mecanicistas y vitalistas, en el marco de unas globalizadas políticas pedagógicas neoliberales (Giroux, 2003; Maturana, 2001; Mujica, 2018, 2019; Prieto, 2018; Toro, 2017). Un ejemplo de ello es la adopción, sin mayor reflexión, de la estandarizada clasificación de las emociones en positivas y negativas (Mujica, 2018; Prieto, 2018). Al respecto, Mujica, Orellana y Canepa (2018) advierten que diferentes estudios en el ámbito pedagógico utilizan dicha clasificación sin hacer referencia con qué aspecto adquirirían dicho valor. Y, precisamente, en aquellos estudios las emociones adquieren el valor en función de la teoría del bienestar subjetivo (Diener, 1994; Diener, Lucas y Oishi, 2018). Es decir, según la dicotomía placer-displacer o agrado-desagrado. Por lo mismo, Giroux (2013) plantea que "cualquier análisis de la pedagogía crítica debe abordar la importancia que juegan los afectos y las emociones en la formación de la identidad individual y la entidad social" (p. 22).

Así, se ha fundamentado que más allá del bienestar subjetivo, y tras un análisis crítico, una misma emoción podría adquirir un valor positivo (favorecer) y negativo (desfavorecer) en relación a aspectos morales y políticos (Scheler, 2005; Mujica, Inostroza y Orellana, 2018; Mujica et al., 2019). En este sentido, emociones como la alegría, el enfado o la vergüenza, pueden contribuir o perjudicar acciones educativas orientadas a la justicia social y la promoción de derechos humanos. Asimismo, se aprecia que una concepción emocional solamente centrada en bienestar subjetivo y, por lo tanto, desmoralizada y despolitizada perjudica la emancipación cultural de las personas. Y, a su vez, propicia la mercantilización de las emociones con una índole fetichista (Manrique, 2015; Monarca, 2016; Scribano, 2010).

Tal como se ha mencionado, la moral y los valores pueden ser interpretados desde diferentes perspectivas $y$, una de ellas, es la del sistema neoliberal, que se 
encuentra muy establecido en el ámbito político-educativo (Apple, 2011; CarterThuillier y Moreno, 2017; Díez, 2010; McLaren, 2015; Oliva y Gascón, 2016). Entre los aspectos negativos para la sociedad que se atribuyen a esta perspectiva sociopolítica, es la interpretación mercantil en torno al derecho humano de la educación y, por consiguiente, la erosión de una verdadera educación integral (Cavieres, 2014; Contreras y Macías, 2002; García-Huidobro, 2007; Mujica e Inostroza, 2020; Nussbaum, 2016; Oliva, 2008; Puga, 2011). O, en palabras de Freire (1975), la concepción bancaria de la educación, que entiende a los educandos como sujetos pasivos que deben almacenar información, de modo que no promueve en ellos una actitud crítica y reflexiva en torno al aprendizaje. Cabe destacar que este modelo se puso a prueba en Chile en un contexto no democrático (Mujica e Inostroza, 2020), y entre sus consecuencias, se encuentra la sistemática privatización de la educación, en desmedro del sistema de educación pública, lo cual ha producido una grave segregación escolar, además de la construcción de un currículo que favorece esta ideología y legitima las injusticias sociales (Plá, 2016).

Dados los sucesos históricos que engloban a este modelo, es evidente que los valores que promueve no son los de la solidaridad con las personas y sectores menos favorecidos, la responsabilidad con el medio ambiente o el respeto por los derechos humanos. Más bien, fomenta algunos de los valores más dañinos a nivel social y ambiental, que son el consumo desmedido y, por tanto, irresponsable. Por consiguiente, también la construcción de una identidad extremadamente individualista o, en otras palabras, egoísta. Una educación fundada en el modelo neoliberal se beneficia de una formación afectiva-social acrítica, caracterizada por la individualización del bienestar y la invisibilización del bienestar social (Manrique, 2015; Mujica, 2018; Silva-Peña y Paz-Maldonado, 2019). Precisamente, porque esto último requiere, inevitablemente, emociones que produzcan malestar subjetivo (Savater, 1997), en beneficio del propio desarrollo ético-moral, de las demás personas, de los demás seres vivos y del medio ambiente en general. Por esa razón, al modelo de la inteligencia emocional (IE), que se encuentra muy próximo a dicha concepción del bienestar subjetivo (BS), se le realiza la siguiente crítica: 
Las emociones, según se deduce de este modelo, se presentan como un fenómeno individual, transparente, intenso e inmediato. No tienen una base aprendida, histórica, cultural, de clase o económica que sea relevante. Son igualmente definidas para todos los seres humanos. Esta abstracta y acrítica definición oculta que el efecto final de la IE no es comprender las propias emociones, sino hacer que el individuo se sienta aceptado, auténtico y perteneciente al grupo. Algo que tiene más que ver con la domesticación y la estandarización (Manrique, 2015, p. 804).

En términos similares se ha planteado que, a nivel laboral, la IE puede ayudar al BS, pero es un modelo útil para la racionalidad neoliberal, que traspasa la completa responsabilidad del BS a nivel individual, desconociendo la responsabilidad del entorno (Gallo, 2013). Por lo tanto, es lógico afirmar que "el nuevo capitalismo demanda y crea redes de interdependencia para las que ordena que ejerzamos nuestras habilidades emocionales para identificarnos con el punto de vista de otros anulando el conflicto social" (Sánchez, 2015, p. 254). En este sentido, Giroux (2013) plantea que el mayor peligro cultural para las nuevas generaciones proviene, en parte, "de sociedades que se niegan a considerar a los niños como una inversión social, que relegan a millones de niños a la pobreza, que reducen el aprendizaje crítico a programas masivos de evaluaciones mecánicas" (p. 23).

Es posible apreciar que en la educación de los países occidentales se encontraría instalado "el reino del "yo mismo" que conlleva, entre otros aspectos, que pensemos que nuestras emociones tienen validez por el simple hecho que las expresamos, que nuestras opiniones (que consideramos propias) tienen validez porque son nuestras" (Sánchez, 2015, p. 254). Así, en los entornos pedagógicos, se promovería la falta de necesidad de que la afectividad sea contrastada con el marco moral y ético que ordena socialización. En consecuencia, por medio de este adiestramiento o estandarización afectiva, que comienza en la educación temprana o infantil (Mujica y Toro, 2019), se está contribuyendo para que las personas sean fácilmente controladas por malos condicionamientos ideológicos y, evidentemente, atrofiando injustificadamente su autonomía (Manrique, 2015). 
De acuerdo con Foucault (2019), las lógicas que configuran la corporalidad, $y$, por ende, las emociones, son apoyadas por los diferentes poderes sociales en función de su conveniencia, ayudándoles al control de la población. En este sentido, no sería casualidad la represión social que existe a ciertas emociones denominadas negativas, como el enfado.

\section{Emociones de rechazo y transformación social}

De acuerdo con Amilburu (2014), la finalidad de la praxis educativa es "la promoción de vidas humanas logradas, facilitando la plena actualización de las potencialidades naturales del sujeto" (p. 233). Por lo tanto, tanto los educadores como los educandos, requieren rechazar todo los aspectos que atenten contra dicha ambiciosa meta, como, por ejemplo, la violencia física, psicológica y sistémica en los entornos educativos. En este sentido, las emociones serían unos de los principales móviles naturales del ser humano, aunque mediadas por factores histórico-culturales, para revelarse a las situaciones interpretadas como injustas e inadecuadas para su desarrollo (Giroux, 2015; Molina y Beltrán, 2007). Prieto (2018) indica que "la ambivalencia emocional, el conflicto, la inquietud e incluso el desasosiego son experiencias que no podemos eliminar de nuestra condición humana sin que esta quede afectada" (p. 313). En concreto, el enfado sería una de las principales respuestas afectivas que retroalimentarían a la persona sobre las situaciones que suscitan dichas emociones. Entonces, por medio de aquella emoción la persona tiene la posibilidad de buscar alternativas para enfrentar $y$, en el mejor de los casos, trascender aquellas problemáticas.

Esta analogía ha quedado bien expresada en la crisis social de Chile en el año 2019, donde la ciudadanía, evidentemente, se ha mostrado enfadada y aburrida de las políticas neoliberales que heredaron de la dictadura militar (Falabella, 2015; Inzunza, Assaél y Scherping, 2011). En efecto, ha sido parte de un movimiento social que exige mayor responsabilidad, justicia y solidaridad en diferentes ámbitos sociales, y por supuesto, en la educación pública. Precisamente, un reciente estudio sobre dicha crisis, indica que "la rabia fue lo que detonó la protesta. La rabia, esa 
emoción que busca la justicia es la que se tomó las calles" (Silva-Peña y PazMaldonado, 2019, p. 200).

En torno al enfado, a nivel cultural, de forma intencionada o no, se han creado diferentes significados que lo asocian, principalmente, a aspectos negativos desde la perspectiva moral. Por ejemplo, se suele asociar a la violencia o al perjuicio de la integridad de las personas (Calvete e Izaskun, 2016; García, Wlodarczyk, Reyes, San Cristóbal y Solar, 2014), de modo que sus aspectos positivos suelen ser poco abordados. Por ello, es preciso aclarar que el enfado, al ser una emoción, es simplemente una respuesta afectiva (Alonso, 2017), de modo que no tiene un valor moral intrínseco (Mujica, 2018; Scheler, 2005; Steinfath, 2014). Por lo tanto, el enfado no se asocia a un solo patrón de conducta, ya que este último variará en función de la configuración subjetiva de cada persona. O sea, el enfado no necesariamente derivará en violencia o en una acción éticamente incorrecta, de modo que, a lo largo de la historia, ha movilizado muchas acciones pacíficas que reivindicaban mejoras en la sociedad.

En función de lo mencionado anteriormente, es fundamental que dicha emoción sea interpretada en función de su neutralidad moral, ya que en cada persona asume un diferente valor moral. En otras palabras, las personas sienten enfado ante diferentes hechos sociales. Tomando como ejemplo los genocidios llevados a cabo en el siglo XX por regímenes totalitarios, es evidente que hubo personas que no sintieron enfado ante tales hechos de crueldad, mientras otras personas percibieron un gran rechazo ante dichos acontecimientos.

En resumen, el enfado expresa el descontento, o la molestia, que se siente ante un aspecto de la realidad y, por lo tanto, tiene un enorme potencial para transformar los aspectos que se interpretan como negativos para la sociedad. Y, las acciones que se emprendan para dicha transformación, dependerán de múltiples factores psicológicos, culturales y morales que median la personalidad humana.

\section{Educar la función moral del enfado}

Sabemos que los procesos educativos no son neutrales, ya que, tanto a nivel formal como informal, la legislación educativa y la cultura reproducen sus 
valoraciones sobre la realidad. Esto mismo ha sido explicado por Dewey (2004), señalando que "la moral es tan amplia como los actos que conciernen a nuestras relaciones con los demás. $\mathrm{Y}$ potencialmente esto incluye todos nuestros actos, aunque no se piense en sus efectos sociales en el momento de su realización" (p. 297). Por consiguiente, el enfado, al ser encarnado por una persona en un contexto concreto, también cumple funciones morales en el acto pedagógico. Cuando se alude a una función moral del enfado, se hace referencia a la capacidad que tendría esa emoción para reflejar el aprehendizaje de ciertos valores y disvalores éticos, como la solidaridad, el egoísmo, la justicia y la injusticia (Mujica, 2019). No obstante, dicha función moral de las emociones tiene una fuerte carga cultural (Elias, 1939; Mauss, 1921; Lévi-Strauss, 2019), de modo que "no es posible estandarizar el valor pedagógico de las emociones, pues cada situación pedagógica debe ser analizada en forma cualitativa, por su significancia moral e histórico-cultural" (Mujica, Orellana y Luis-Pascual, 2019, p. 85).

Un estudio logró reconocer que el enfado era uno de los principales recursos que utilizaba el profesorado para lograr un clima propicio para el aprendizaje (Funes, 2017). Dicha investigación le otorga, implícitamente, un carácter negativo a esa emoción encarnada en la práctica docente, ya que sería parte importante de una relación pedagógica incorrecta con los educandos, por desarrollarse en un marco ideológico de excesiva verticalidad pedagógica. En concreto, dicha valoración del enfado del profesorado es oportuna, ya que el abuso del enfado por parte de los educadores puede provocar un contexto marcado por el autoritarismo, sobre todo, cuando existe un currículo tecnocrático y al servicio del mercado. Sin embargo, el enfado del profesorado, y del alumnado, no puede ser visto, en todas las circunstancias, como algo negativo en el aula, ya que dicha emoción le permitirá expresar su rechazo a múltiples situaciones que ameritan ser criticadas. Por ejemplo, como sucedió el año 2019 en Palma de Mallorca (Bohorquéz, 2019), si un grupo de estudiantes se organiza y realiza un video promoviendo la ideología nazi, el enfado del profesorado de dicho centro educativo estaría totalmente justificado. Y, más aún, la sanción al profesorado que acepta dichas acciones sería totalmente 
necesaria, como se realizó con el docente que se hizo parte del mencionado saludo nazi en España.

Comprendiendo el importante rol que tiene el enfado en la formación moral de las personas, es preciso promover un discurso pedagógico que enfrente el sesgo hedonista, vitalista y neoliberal que ignora la legitimidad de encarnar afectivamente el rechazo a las situaciones valoradas en forma negativa. Así, Mujica y Toro (2019), han planteado la necesidad de evitar la interpretación cerrada de las emociones en torno al bienestar subjetivo, debido a que se deben considerar aspectos intersubjetivos y transubjetivos. De esta forma, la perspectiva del bienestar subjetivo dejaría de ser un discurso hegemónico que niega las emociones que pueden producir malestar subjetivo, como es el caso del enfado. En este sentido, es fundamental comprender que el bienestar subjetivo ha sido muy importante en las relaciones humanas y, por supuesto, en los procesos educativos. Al respecto, Giroux (2003) plantea que, en el marco de una interpretación crítica de la pedagogía, es relevante "entender cómo la producción de significado está atada a inversiones emocionales y a la producción de placer" (p. 212).

En resumen, el enfado, al igual que las otras emociones, pueden contribuir a la mejora de la sociedad y, principalmente, en términos de justicia social y derechos humanos. $\mathrm{Y}$, por cierto, esta idea viene siendo respaldada en diferentes investigaciones documentales y empíricas sobre la materia (Azzarito, Solmon y Harrisson, 2006; Iriarte e Ibarrola-García, 2010; McCaughtry, Barnard, Martin, Shen y Hodges, 2006; Molina y Beltrán, 2007; Mujica y Toro, 2019; Silva-Peña y PazMaldonado, 2019). En suma, en los entornos educativos se debe trascender la desmoralización de las emociones y, claramente, del enfado, reconociendo que "existe una estrecha relación entre la experiencia afectiva y la vida moral" (Prieto, 2018, 313). Igualmente, es muy beneficioso para la comprensión de las relaciones humanas que, desde una perspectiva crítica, se desarrollen investigaciones que desvelen los significados implícitos en el enfado que experimentan las personas en la praxis educativa. De este modo, se podrían dirigir investigaciones orientadas al ámbito pedagógico informal que indaguen los significados emocionales en el ámbito familiar y en los entornos comunitarios. En la educación formal, es posible 
desarrollar estudios en los diferentes niveles académicos en función de múltiples factores, por ejemplo, el género o las diferentes disciplinas académicas. De este modo se lograría visibilizar las experiencias de educadores y educandos. También en el caso de las instituciones de educación formal, se podrían interpretar las experiencias de las personas encargadas de dirigirlas, es decir, de los equipos directivos.

\section{Conclusiones}

Se concluye que, dada la contraposición con el bienestar subjetivo y la concepción bancaria de la educación, el enfado es una emoción que ha sido dotada, principalmente, de un carácter negativo en los discursos pedagógicos. Así, se ha invisibilizado su positivo potencial para la formación moral de las personas y, en consecuencia, para la mejora de la sociedad. Indudablemente, el enfado ha logrado ser protagonista en múltiples reivindicaciones en torno a diferentes ámbitos sociales, entre ellos, la educación. La educación pública es la que se ha encontrado más amenazada en el contexto neoliberal, por lo que muchas de esas reivindicaciones han desafiado dicho modelo que intenta dejarla al servicio del mercado y no del desarrollo pleno de las personas. Precisamente, destacan los movimientos sociales que han existido en los últimos años en Latinoamérica y, sobre todo, en Chile, cuna del neoliberalismo (Campos-Martínez y Guerrero, 2016; Mujica e Inostroza, 2020; Silva-Peña y Paz-Maldonado, 2019).

Se considera muy oportuno que, tanto en la educación informal como en la formal, se asuma una perspectiva crítica sobre el rol que tiene el enfado en la emancipación de las personas (Mujica y Toro, 2019; Silva-Peña y Paz-Maldonado, 2019). De esta forma, entendiendo que la educación debe promover un desarrollo pleno o integral de cada persona (Savater, 1997), se podrá comprender que el enfado es tan importante como la alegría, ya que ambas emociones contribuyen a percibirse, valorarse y transformarse en función del marco moral que se estime más conveniente. Además, dicha emoción ayuda a percibir, valorar y transformar, los aspectos que se estimen convenientes de la sociedad y, sobre todo, los presentes en el contexto neoliberal. En cuanto a las estimaciones que realiza cada persona en 
función de su enfado sobre un tema sociocultural, idealmente desde una perspectiva pedagógica crítica, es fundamental que los educandos puedan conocer, comprender, reflexionar y debatir el fondo de las diferentes ideologías que sustentan dicha temática. En este sentido, no se debe reducir la enseñanza en temas sociales a la legalidad vigente, aunque exista consenso sobre su fundamentación, ya que, para comprender el fondo de las estimaciones morales hegemónicas en la sociedad, es fundamental cuestionarlas. Además, la memoria histórica nos recuerda que las ideas que en algún momento fueron interpretadas como justas y adecuadas, por medio de la crítica social y, evidentemente, del enfado con el orden ideológico establecido, pudieron ser trascendidas.

\section{Referencias bibliográficas}

Alonso, J. (2017). Las emociones. La base neurológica del comportamiento. Barcelona: RBA.

Amilburu, G. (2014). Filosofía y actitud filosófica: sus aportaciones a la educación. Revista Española de Pedagogía, 72(258), 231-247.

Apple, M. (2011). Democratic education in neoliberal and neoconservative times. International Studies in Sociology of Education, 21(1), 21-31. doi: $10.1080 / 09620214.2011 .543850$

Azzarito, L., Solmon, M. y Harrison, L. (2006). "...If I Had a Choice, I Would....". A Feminist Poststructuralist Perspective on Girls in Physical Education. Research Quarterly for Exercise and Sport, 77(2), 222-239. doi: 10.1080/02701367.2006.10599356

Bohorquéz, L. (2019). Sancionado un profesor por fotografiar a sus alumnos haciendo el saludo nazi. Periódico El País. Disponible en https://bit.ly/2VD9pBE Calvete, E. y Izaskun, O. (2016). Violencia Filio-Parental: frecuencia y razones para las agresiones contra padres y madres. Psicología Conductual, 24(3), 481-495. doi: $10.2307 /$ j.ctvrzgzmg.7 
Campos-Martínez, J. y Guerrero, P. (2016). Efectos indeseados de la medición de la calidad educativa en Chile. La respuesta de la sociedad civil. Cadernos Cedes,36(100), 355-374. doi: 10.1590/cc0101-32622016171351

Casado, C. y Colomo, R. (2006). Un breve recorrido por la concepción de las emociones en la Filosofía Occidental. A Parte Rei, 47, 1-10.

Dewey, J. (2004). Democracia y educación (6a ed.). Madrid: Morata.

Diener, E. (1994). Assessing subjective well-being: Progress and opportunities. Social Indicators Research, 31, 103-157. doi: 10.1007/bf01207052

Diener, E., Lucas, R. y Oishi, S. (2018). Advances and open questions in the science of subjective well-being. Collabra: Psychology, 4(1), 1-49. doi: 10.1525/collabra.115

Díez, E. (2010). La globalización neoliberal y sus repercusiones en educación. Revista electrónica interuniversitaria de formación del profesorado, 13(2), 23-38. Durán, L. J. (2011). ¿El deporte transmite valores? En A. Madariaga y J. Cuencua (Eds.), Los valores del ocio: cambio, choque e innovación (pp. 161-178). Bilbao: Universidad de Deusto.

Elias, N. (1939). Über den Prozeß der Zivilisation. Basen: Verlag Haus zum Falken. Falabella, A. (2015). El mercado escolar en Chile y el surgimiento de la nueva gestión pública: El tejido de la política entre la dictadura neoliberal y los gobiernos de la centroizquierda (1979 a 2009). Educação \& Sociedade, 36(132), 699-722. doi: 10.1590/es0101-73302015152420

Foucault, M. (2019). Microfísica del poder. Buenos Aires: Siglo veintiuno.

Freire, P. (1975). Pedagogía del Oprimido. Madrid: Siglo XXI.

Funes, S. (2017). Las emociones en el profesorado: el afecto y el enfado como recursos para el disciplinamiento. Educação e Pesquisa, 43(3), 785-798. doi: 10.1590/s1517-9702201610149719

García, F., Wlodarczyk, A., Reyes, A., San Cristóbal, C. y Solar, C. (2014). Violencia en la pareja, apoyo social y bienestar psicológico en adultos jóvenes. Revista Ajayu, 12(2), 246-265.

Giroux, H. (2003). Pedagogía y Política de la Esperanza. Teoría, cultura y enseñanza. Una antología crítica. Buenos Aires: Amorrortu. 
Giroux, H. (2013). La Pedagogía crítica en tiempos oscuros. Praxis Educativa, 1712), $13-26$.

Giroux, H. (2015). Cambiando el guión: repensando la resistencia de la clase obrera. Revista Internacional de Educación para la Justicia Social, 4(2), 100-107. doi: 10.15366/riejs2015.4.2

Inzunza, J., Assaél, J. y Scherping, G. (2011). Formación docente inicial y en servicio en Chile. Tensiones de un modelo neoliberal. Revista Mexicana de Investigación Educativa, 16(48), 267-292.

Iriarte, C. e Ibarrola-García, S. (2010). Revisión de estudios sobre la vivencia emocional de la discapacidad intelectual por parte de los hermanos. Estudios Sobre Educación, 19, 53-75.

Lévi-Strauss, C. (2019). Raza y cultura (9a ed.). Madrid: Cátedra.

Maturana, H. (2001). Emociones y lenguaje en educación y política. Santiago de Chile: Dolmen.

Mauss, M. (1921). L'expression obligatoire des sentiments (Rituels oraux funéraires australiens). Journal de Psychologie, 18, 425-434.

McCaughtry, N., Barnard, S., Martin, J., Shen, B. y Hodges, P. (2006). Teachers' perspectives on the challenges of teaching physical education in urban schools. Research Quarterly for Exercise and Sport, 774), 486-497. doi: 10.1080/02701367.2006.10599383

McLaren, P. (2015). Pedagogía crítica y lucha de clases en la era del terror neoliberal. Revista Internacional de Educación para la Justicia Social, 4(2), 2966. doi: 10.15366/riejs2015.4.2

Molina, J. P. y Beltrán, V. J. (2007). Incompetencia motriz e ideología del rendimiento en Educación Física: El caso de un alumno con discapacidad intelectual. Motricidad. European Journal of Human Movement, 19, 165-190.

Monarca, H. (2016). Sobre educación, política y sociedad. Revista Educación, Política y Sociedad, 1(1), 4-9.

Mujica, F. (2018). Educar y suscitar emociones en la educación: análisis crítico de su contribución al desarrollo moral. Ensayos. Revista de la Facultad de Educación de Albacete, 33(2), 15-27. doi: 10.18239/ensayos.v33i2.1540 
Mujica, F. (2019). Suscitar buenas emociones en la educación formal: Análisis según la ética de Max Scheler. Revista Dilemas Contemporáneos: Educación, Política y Valores, 6(3), 1-15.

Mujica, F., Inostroza, C. y Orellana, N. (2018). Educar las emociones con un sentido pedagógico: un aporte a la justicia social. RIEJS. Revista internacional de Educación para la Justicia Social, 72 ), 113-127. doi: 10.15366/riejs2018.7.2.007

Mujica, F. e Inostroza, C. (2020). Políticas educativas, evaluaciones estandarizadas y formación escolar integral en Chile: análisis de la desvalorización a diferentes materias del currículo. Dilemas Contemporáneos: Educación, Política y Valores, ス2), 1-20.

Mujica, F., Orellana, N. y Canepa, P. (2018). Educación emocional en la asignatura de educación física: análisis crítico del valor positivo o negativo de las emociones. Revista Dilemas contemporáneos: educación, política y valores, 6(1), 1-23.

Mujica, F., Orellana, N. y Luis-Pascual, J.C. (2019). Perspectiva moral de las emociones en los contextos de educación formal. Revista Ensayos Pedagógicos, 14(1), 69-90. doi: 10.15359/rep.14-1.4

Mujica, F. y Toro, M. (2019). Formación afectiva en la Educación Parvularia de Chile. Cuadernos de Investigación Educativa, 10(2), 57-71. doi: 10.18861/cied.2019.10.2.2907 Nussbaum, M. (2016). Educación para el lucro, educación para la libertad. Nomadas, 44, 13-25. doi: 10.30578/nomadas.n44a1

Prieto, M. (2018). La psicologización de la educación: implicaciones pedagógicas de la inteligencia emocional y la psicología positiva. Educación XX1, 21(1), 303-320. doi: $10.5944 /$ educXX1.16058

Puig, N. (2012). Emociones en el deporte y sociología. Revista Internacional de Ciencias del Deporte, 8(28), 106-108.

Savater, F. (1997). El valor de educar. Barcelona: Ariel.

Scribano, A. (2010). Cuerpo, emociones y teoría social clásica: hacia una sociología del conocimiento de los estudios sociales de los cuerpos y las emociones. En J. Grosso y M. Boito (Eds.), Cuerpos y Emociones desde América Latina (pp. 15-38). Córdova: CEA-CONICET y Universidad Nacional de Catamarca.

Scheler, M. (2005). Esencia y formas de la simpatía. Sígueme: Salamanca. 
Scheler, M. (2010). Amor y conocimiento y otros escritos. Madrid: Palabra.

Silva-Peña, I. y Paz-Maldonado, E. (2019). Formación docente para la justicia social desde la perspectiva emocional: indagaciones narrativas en el contexto de la revolución del torniquete. Revista Interuniversitaria de Formación del Profesorado, 94(33.3), 195-212.

Steinfath, H. (2014). Emociones, valores y moral. Universitas Philosophica, 31(63), 71-96. doi: 10.11144/javeriana.uph31-63.evms

Zaccagnini, J. (2008). La comprensión de la emoción. Una perspectiva psicológica. En M. Jiménez (Ed.), Educación Emocional y Convivencia en el Aula (pp.31-84). Madrid: Ministerio de Educación, Política Social y Deporte.

Fecha de recepción: 13 de enero de 2020

Fecha de aceptación: 27 de abril de 2020 